\title{
High-Pressure Studies of Ordering Phenomena in Heavy-Fermion Compounds
}

\author{
J.J.M. Franse, Amsterdam
}

(Natuurkundig Laboratorium der Universiteit van Amsterdam)

The discovery of a new class of intermetallic compounds with exceptional low-temperature properties, the heavyfermion systems, has generated a lot of excitement amongst solid-state physicists, both theoreticians and experimentalists. Experimentalists are faced with anomalies in thermodynamic and transport properties at temperatures typically below about $10 \mathrm{~K}$. These anomalies indicate an effective mass of the electrons at the Fermi level, which is two orders of magnitude larger than the free-electron mass. Although this large effective mass is intriguing in itself, even more astonishing are the occurrence of superconductivity in the heavy-fermion compounds and the extremely large specificheat anomalies at the superconducting transition temperature, indicating that heavy-mass electrons participate in the superconductivity. These features have given rise to suggestions from theoreticians of an exotic type of pair bonding between the electrons.

In the familiar models of superconductivity, the necessary attractive interaction between two electrons is provided by electron-phonon processes. The most favourable situation occurs when the orbital function of the electron pair is symmetrical with spins opposite (swave or singlet pairing). In these approaches, use is made of the fact that the Debye temperature, representing the range of phonon energies, is much smaller than the Fermi temperature; in normal metals, the Fermi temperature is of the order of $10^{4}$ to $10^{5} \mathrm{~K}$ whereas the Debye temperature is a few hundred kelvin. In the heavy-fermion compounds however, as the Fermi temperature is inversely proportional to the electronic mass, its value is reduced to a value comparable to the Debye temperature. In this situation, theoreticians have been looking for other bonding mechanisms and at the present time a good candidate is an attractive interaction intermediated by so-called spin fiuctuations.

In this approach, the bonding of an electron pair is connected with the spin polarisation of the electron gas that arises from the magnetic interactions. These interactions dress the electron with a cloud of polarised spins and it is this dressing that is at the origin of the high effective mass of the electrons. One conjecture is that the most favourable pair bonding in this case occurs for electrons with parallel spins and hence with an antisymmetric orbital function (p-wave or triplet pairing). The energy range of the spin fluctuations is represented by the spin-fluctuation temperature, $T_{\mathrm{SF}}$, which appears to be of the order of $10 \mathrm{~K}$, thus small compared to the effective Fermi temperature. Since in this picture magnetic interactions play an important role, a competition between superconductivity and magnetic order is to be expected.

A somewhat arbitrary limit for characterising a compound as a heavy-fermion system is a value of $400 \mathrm{~mJ} / \mathrm{K}^{2} \mathrm{~mol}$ for the electronic coefficient $\gamma$ of the linear term in the specific heat temperature relation. (In normal metals the corresponding figure is between 1 and 10). A number of compounds are presently known to meet this criterion: $\mathrm{CeAl}_{3}$, $\mathrm{CeCu}_{2} \mathrm{Si}_{2}, \mathrm{CeCu}_{6}, \mathrm{UBe}_{13}, \mathrm{UPt}_{3}, \mathrm{U}_{2} \mathrm{Zn}_{17}$ and $\mathrm{UCd}_{11}$. Three of them become superconducting below $1 \mathrm{~K}\left(\mathrm{CeCu}_{2} \mathrm{Si}_{2}\right.$, $\mathrm{UBe}_{13}$ and $\mathrm{UPt}_{3}$ ), two exhibit antiferromagnetic order below $10 \mathrm{~K}\left(\mathrm{U}_{2} \mathrm{Zn}_{17}\right.$ and $U \mathrm{Cd}_{11}$ ) and the remaining two show no order at all down to the millikelvin temperature range $\left(\mathrm{CeAl}_{3}\right.$ and $\mathrm{CeCu}_{6}$ ).

Superconductivity and magnetic order in the heavy-fermion systems are sometimes extremely sensitive to deviations from stoichiometry, to chemical purity, to the atomic order or to the state of stress. In $\mathrm{CeCu}_{2} \mathrm{Si}_{2}$ compounds which are copper-rich, superconductivity is enhanced, whereas in $\mathrm{UPt}_{3}$, the superconducting temperature, $T_{S^{\prime}}$ is strongly dependent on annealing procedures. Besides that, superconductivity in $\mathrm{UPt}_{3}$ is easily destroyed by powdering the material or by substituting only 0.5 at $\% \mathrm{Pt}$ by iso-electronic Pd. On further increasing the palladium content in $\mathrm{UPt}_{3}$, the heavy-fermion regime subsists up to 10 at $\%$, the alloys first showing spin-fluctuation effects and then, at between 1 and 2 at $\% \mathrm{Pd}$, an onset to antiferromagnetic order with maximal values for the Néel temperature of about $6 \mathrm{~K}$ around 5 at $\% \mathrm{Pd}$.

In this paper we are dealing with highpressure studies of these ordering phenomena in the heavy-fermion compounds. Successively we discuss the experimental approaches and the pressure effects on spin fluctuations, superconductivity and antiferromagnetic order. For an introduction into the physical properties of the heavy-fermion compounds we refer to a review paper by Stewart ${ }^{1}$ ).

\section{Experimental Approaches}

Since ordering in the heavy-fermion systems turns out to be such a delicate feature that is easily destroyed or enhanced, a variation of the external parameters (uniaxial stress, hydrostatic pressure, chemical pressure induced by substitutions) can be expected to affect markedly the phenomena. Strong anisotropies in some of these f-electron compounds make the application of uniaxial stress along certain crystallographic directions an especially appropriate tool, although such experiments have not yet been performed. Chemical substitutions and hydrostatic pressures, however, have been applied to poly- and monocrystalline samples and have been found to create conditions that span the range from Kondo behaviour (resistivity decreasing with increasing temperature over a certain temperature range, as observed in $\mathrm{CeCu}_{2} \mathrm{Si}_{2}$ and $\mathrm{UBe}_{13}$ ) to spin-fluctuation phenomena (resistivity proportional to $T^{2}$ at low temperatures and saturating when approaching room temperature, as observed in $\mathrm{UPt}_{3}$ ).

In order to study the pressure dependence of thermodynamic quantities it is not always necessary to employ high mechanical forces which are often damaging to the sample, particularly in the $10 \mathrm{GPa}$ (100 kbar) pressure range where the pressure is transmitted via solid materials. Experiments in this pressure range are mostly confined to transport properties and X-ray studies. Pressurising through a gas or fluid medium limits experiments in general to 1 to $2 \mathrm{GPa}$, but especially when using a helium medium, hydrostatic conditions can be preserved 
rather well even at low temperatures, making the study of thermal and magnetic properties on single crystal samples feasible without damage to the sample. Nevertheless, if one is interested in the initial pressure dependences of, for instance, the susceptibility or the specific heat, it is equally possible to perform respectively forced magnetostriction or thermal expansion measurements. On comparing these with the high-pressure experiments as such, we can check the reliability of our information. These different methods have been successfully applied to $\mathrm{UPt}_{3}$ by De Visser ${ }^{2}$ ) using single crystals which are imperative for such an investigation on a non-cubic material.

\section{Spin Fluctuations}

Increasing pressure depresses the low-temperature anomalies that are characteristic for the heavy-fermion behaviour. This depression is illustrated for $\mathrm{UPt}_{3}$ in Figs. 1 and 2 which show the temperature dependence of the resistivity and the specific heat as a function of pressure. Both the coefficient $A$ of the $T^{2}$-term in the resistivity and the coefficient $\gamma$ of the linear term in the specific heat temperature relation decrease with pressure. The parameter $A / \gamma^{2}$ turns out to be almost pressure independent and its value is found to be close to $1.0 \times$

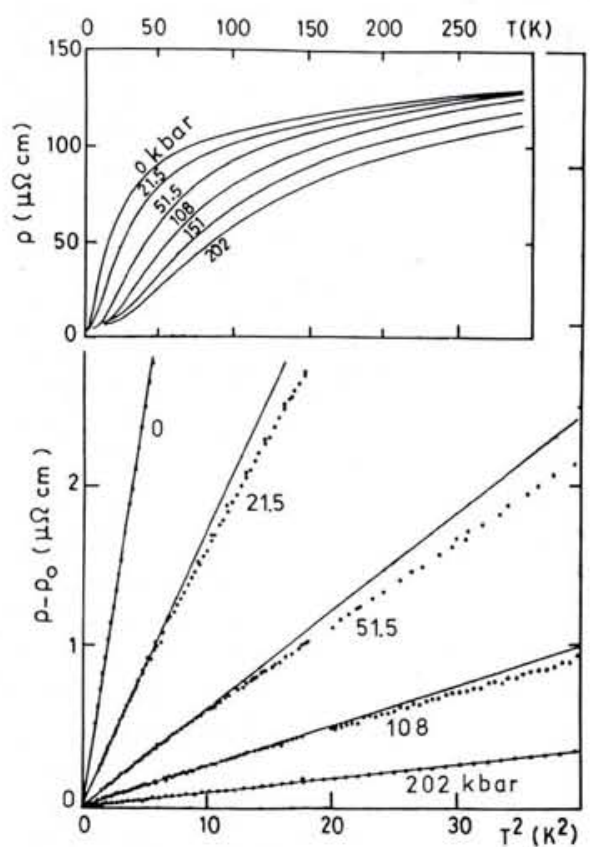

Fig. 1 - High-pressure resistivity data of monocrystalline $\mathrm{UPt}_{3}$ whiskers taken from Ponchet et al. 3); at low temperatures the experimental data follow the relation $\rho(T)=$ $\rho_{o}+A T^{2}$, with $\rho_{0}$ the residual resistivity in the normal state; the negative pressure dependence of the coefficient $A$ of the $T^{2}$-term in the resistivity points to a strong increase of the spin-fluctuation temperature with pressure.

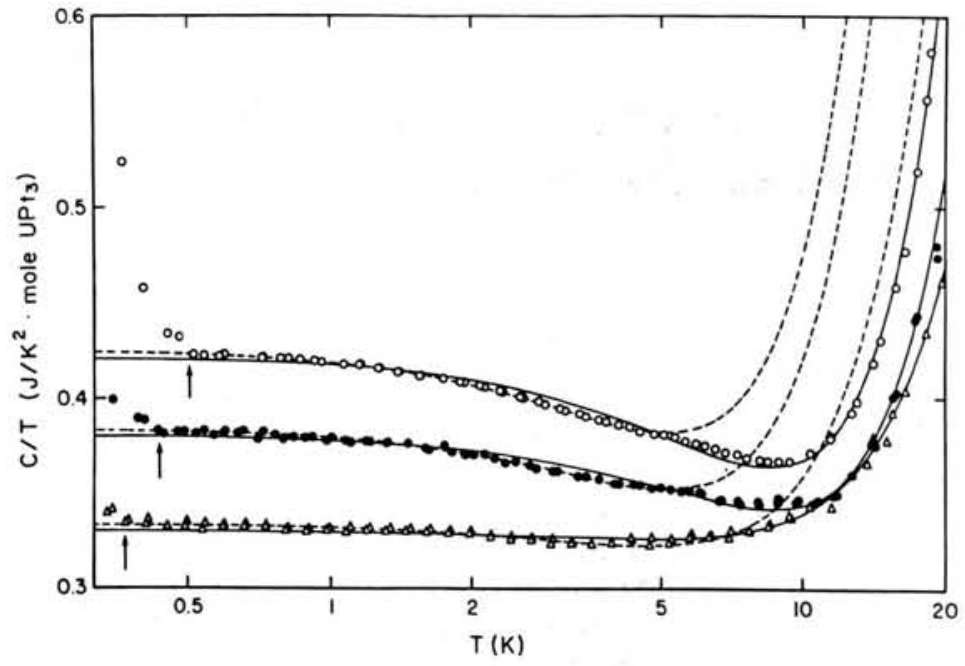

Fig. 2 - Pressure dependence of the specific heat of UPt ${ }_{3}$ as reported by Brodale et al. $\left.{ }^{4}\right)$; data have been taken at 1 bar (o), $3.8 \mathrm{kbar}(\mathrm{O})$ and $8.9 \mathrm{kbar}(\Delta)$; the experimental data in the normal state have been fitted to the expression $c / T=\gamma+\varepsilon T^{2}+\delta T^{3} \ln T(K)$ for temperatures below $4 \mathrm{~K}$ (dashed curves) and to temperatures below $20 \mathrm{~K}$ (solid curves); transitions to the superconducting state are indicated by small arrows.

$10^{-5} \mu \Omega \mathrm{cm}$ (mole $\left.\mathrm{K} / \mathrm{mJ}\right)^{2}$, a common value for nearly all heavy-fermion compounds ${ }^{5}$ ). This universal result enhances the challenge to bring these systems into the same theoretical framework. Working within the concept of spin fluctuations, the parameter $A$ is found to be inversely proportional to the square of the spin-fluctuation temperature. According to the experimental observations for $\mathrm{UPt}_{3}$, the coefficient $\gamma$ varies with pressure as $1 / T_{\mathrm{SF}}$. Pressure effects on the susceptibility of UPt ${ }_{3}$ can also be expressed in terms of a pressure-dependent spin-fluctuation temperature. $\mathrm{Nu}$ merical results for the relative pressure dependence of $T_{\mathrm{SF}}$ as determined from resistivity and susceptibility measurements under pressure, coincide within the experimental error. Although this feature cannot be considered as a proof that spin fluctuations are at the bottom of the heavy-fermion behaviour fo this particular compound, it shows at least that the model is consistent with the experimental observations.

\section{Superconductivity}

Both negative and positive pressure effects have been reported for the superconducting transition temperature in heavy-fermion compounds. For example, in the uranium-based heavy-fermion superconductors, $\mathrm{UBe}_{13}$ and $\mathrm{UPt}_{3}$, the pressure dependence of $T$ is moderately negative and comparable to what is usually observed for a conventional type of superconductor, see Fig. 2 and references 6 and 7 . The most interesting feature, however, is not the sign or absolute value so much as the relation between pressure effects in the normal and superconducting states. Studies of the pressure dependence of specific heat and resistivity can provide us with the required information on such normalstate properties like the heavy electronic mass and the Kondo or spin-fluctuation temperatures, but interpretation of the data depends strongly on appropriate models which are not always available; the temperature dependences that are observed in these materials are often complex.

Since spin fluctuations are known to suppress superconductivity in normal metals, the coexistence of both phenomena in $\mathrm{UPt}_{3}$ was certainly not expected. A start to interpreting the superconductivity and its pressure dependence for this compound has been made by Pethick et al. ${ }^{8}$ ). Working from a Fermi liquid model, the normal and superconducting state parameters are expressed in terms of the same Landau parameters. Values for these parameters derived in the normal state indicate a $p$-wave-type of superconductivity and predict a satisfactory correspondence with the experimentally observed pressure dependence of the superconducting transition temperature. This promising approach is applicable to UPt $\mathrm{UP}_{3}$ since an adequate fit of the specific heat data is given by Fermi liquid theory that includes a $T^{3}$ In $T(\mathrm{~K})$ term, see Fig. 2. A similar description of the other heavy-fermion superconductors is lacking at present.

\section{Antiferromagnetic Ordering}

Antiferromagnetic order in the heavyfermion systems has been observed for $\mathrm{U}_{2} \mathrm{Zn}_{17}$ and $\mathrm{UCd}_{11}$, with values for the Néel temperature, $T_{N}$, of 9.7 and $5.0 \mathrm{~K}$, respectively. Although in $\mathrm{UPt}_{3}$ no longrange magnetic order has been found, 
high-field magnetisation measurements show a metamagnetic-type of transition in this material around $20 \mathrm{~T}$ in the liquidhelium temperature region, reminiscent of some type of antiferromagnetism ${ }^{9}$ ). Upon substituting a few percent of $\mathrm{Pt}$ by $\mathrm{Pd}$ or $\mathrm{U}$ by $\mathrm{Th}$, this latent antiferromagnetism becomes apparent below an ordering temperature of about $6 \mathrm{~K}^{10,11}$ ). Experiments indicate that antiferromagnetism in these alloys is associated with Fermi surface instabilities (spindensity waves). High-pressure experiments reveal a strong depression of $T_{N}$ in $\mathrm{U}(\mathrm{Pt}, \mathrm{Pd})_{3}$ compounds $\left.{ }^{12}\right)$ in contrast to $\mathrm{U}_{2} \mathrm{Zn}_{17}$ and $\mathrm{UCd_{11 }}$, where small and positive pressure effects have been reported ${ }^{13}$ ). In these latter cases, the positive pressure effects are claimed to result from competing Kondo and indirect exchange interactions.

\section{Basic Mechanisms}

It seems tempting to ascribe the large substitution effects on ordering phenomena in the heavy-fermion compounds either to impurity scattering, to lattice spacing effects or to critical changes in $5 f$-electron localisation or hybridisation between the uranium and surrounding ligand states. Taking these in turn, in our experience, impurity scattering is not the dominant parameter in suppressing superconductivity in the $(\mathrm{U}, \mathrm{Th})(\mathrm{Pt}, \mathrm{Pd})_{3}$ system. Unannealed UPt ${ }_{3}$ samples with $\rho(300 \mathrm{~K}) / \rho_{0}$-values of 30 are superconducting below $0.3 \mathrm{~K}$, whereas in a 1 at $\%$ $\mathrm{Pd}$ sample with the same value for this resistivity ratio, no superconductivity has been observed down to $40 \mathrm{mK}$. Because the molar volume of $\mathrm{UPt}_{3}$ increases with increasing thorium, but decreases with increasing palladium content, the atomic volume cannot be the decisive parameter for the transition from the superconducting to the magnetically ordered state in the $(\mathrm{U}, \mathrm{Th})(\mathrm{Pt}, \mathrm{Pd})_{3}$ system either.

Further parameters to be considered are the anisotropies in the lattice constants. The c/a ratio for the hexagonal compound $\mathrm{UPt}_{3}$ increases on substituting with thorium or palladium. This suggests that the depression of superconductivity and promotion of antiferromagnetic order goes with decreasing $c / a$ values, and since this ratio increases with pressure (the compressibility is slightly anisotropic in $\mathrm{UPt}_{3}$ ), the conclusion is consistent with the results of the high-pressure studies on the antiferromagnetic ordering temperature. However, it does not explain the depression of superconductivity by both high pressure and increasing thorium or palladium content. Substitutions on the uranium
Fig. 3 - Phase diagram of the $U(P t, P d)_{3}$ system, showing the composition ranges in which superconductivity (S) and antiferromagnetic order (AF) are observed as well as the occurrence of spin-fluctuation effects (SF) and Kondo-type of behaviour (K); the compounds with a palladium concentration up to 10 at \% all exhibit large effective electron masses at low temperatures with maximum values around 10 at \%; by the application of pressure, the ordering temperatures $\left(T_{S}\right.$ and $\left.T_{N}\right)$ are depressed whereas the spin-

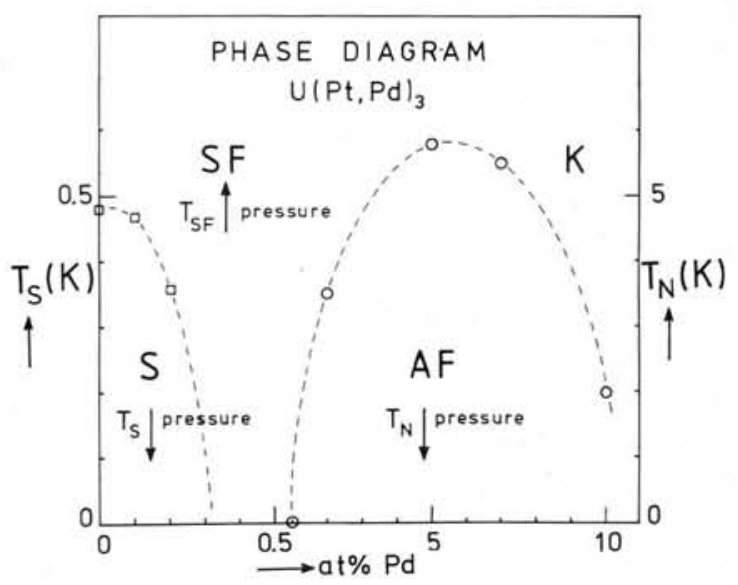

fluctuation temperature $\left(T_{S F}\right)$ is shifted to higher values. sublattice (thorium) as well as on the platinum sublattice (palladium) most probably lead to increasing localisation of the $5 f$ electrons due to increased $\mathrm{U}-\mathrm{U}$ distances or to a reduced $\mathrm{f}-\mathrm{d}$ hybridisation, respectively. This increased localisation is undoubtedly in favour of a magnetically ordered state and compatible with the negative pressure effect on $T_{N}$. But the negative pressure effect on $T_{\mathrm{S}}$, again remains unexplained. Clearly our present understanding of the mechanisms that drive the (U,Th) $(\mathrm{Pt}, \mathrm{Pd})_{3} \mathrm{com}$ pounds from the superconducting state into the magnetically ordered (spindensity-wave) state is still poor.

\section{Conclusion}

To conclude this examination of pressure effects in the heavy-fermion systems, a tentative phase diagram of ordering phenomena in the pseudo-binary compound $\mathrm{U}(\mathrm{Pt}, \mathrm{Pd})_{3}$ is presented in Fig. 3. Up to a palladium concentration of 10 percent, these compounds remain in the heavy-fermion regime with values of the electronic-mass enhancement of 180 or more. Within this range, the low-temperature anomalies in the resistivity change from spin-fluctuation-type to Kondo-type $\left.{ }^{14}\right)$. The ordering phenomena change from superconductivity to antiferromagnetic order within a narrow

\section{Professorship in Norway: Theoretical solid state physics}

The Norwegian Research Council for Science and the Humanities (NAVF) announces a full professorship in theoretical solid state physics, to be located either at The University of Oslo, or at The Norwegian Institute of Technology, Trondheim. (Decision on the location will be made soon.) After an initial period of about 3 years under the Council, the position will be tenured by the host university.

It is the purpose of the Council to strengthen considerably the Norwegian condensed matter research effort. To this end the Council has created a 5 -year programme for materials sciences. The new professorship is to be seen in this context.

The professor should possess broad qualifications in solid state theory, and be well versed in modern topics in this field. He or she is expected to conduct active research in solid state theory, teach advanced solid state courses, supervise the work of students and research fellows, and be active in maintaining and strengthening collaboration between scientists in Norway and foreign countries. Experience and interest in cooperation with experimentalists will be highly valued.

The appointment is from 1 September 1987, but a later date can be arranged if necessary. Those interested in the appointment should send a curriculum vitae, a list of publications and the names of three referees before 15 May 1987 to the

Director Natural Sciences

The Norwegian Research Council for Science and the Humanities

Sandakerveien 99

0483 Oslo 4

Norway

Those wishing to inquire about the position or to recommend suitable candidates are urged to contact the Director at the above address, or by phone: $+47-2-157012$. ext. 155

RMF $25 / 87$ 
concentration range around 1 at $\% \mathrm{Pd}$ where no ordering has been observed. The spin-fluctuation temperature increases with the application of pressure, whereas the superconducting and antiferromagnetic ordering temperatures are depressed, leaving open the possibility that the same interactions are responsible for superconductivity and antiferromagnetism.

\section{REFERENCES}

1. Stewart G.R., Rev. Mod. Phys. 56 (1984) 755.

2. De Visser A., Thesis University of Amsterdam (1986).

3. Ponchet A., Mignot J.M., de Visser A., Franse J.J.M. and Menovsky A., J. Magn. Magn. Mat. 54-57 (1986) 399.

4. Brodale G.E., Fisher R.A., Philips N.E., Stewart G.R. and Giorgi A.L., Phys. Rev. Lett. 57 (1986) 234.

5. Kadowaki K. and Woods S.B., Solid State Commun. 58 (1986) 507.

6. Chen J.W., Lambert S.E., Maple M.B., Fisk Z., Smith J.L. and Ott H.R. in: Proc. LT-17 (North-Holland, Amsterdam) Part 1, 1984, p. 325.

7. Willis J.O., Thompson J.D., Fisk Z., de Visser A., Franse J.J.M. and Menovsky A. Phys. Rev. B 31 (1985) 1654.

8. Pethick C.J., Pines D., Quader K.F., Bedell K.S. and Brown G.E., Phys. Rev. Lett. 57 (1986) 1955.

9. Franse J.J.M., Frings P.H., de Visser A. Menovsky A., Palstra T.T.M., Kes P.H. and Mydosh J.A., Physica 126B (1984) 116.

10. De Visser A., Klaasse J.C.P., van Sprang M., Franse J.J.M., Menovsky A., Palstra T.T.M. and Dirkmaat A.J., Phys. Lett. 113A (1986) 489.

11. Ramirez A.P., Batlogg B., Cooper A.S. and Bucher E., Phys. Rev. Lett. 57 (1986) 1072.

12. Van Sprang M., de Visser A., Franse J.J.M., Menovsky A. and Dirkmaat A.J., J. Magn. Magn. Mat., in print.

13. Thompson J.D., Fisk Z. and Ott H.R., J. Magn. Magn. Mat. 51 (1986) 141.

14. Verhoef R., de Visser A., Menovsky A., Riemersma A.J. and Franse J.J.M., Physica 142B (1986) 11.

\section{CMD ELECTIONS}

\section{Call for Nominations for the Sections of the Condensed Matter Division}

Elections to the Committees responsible for the Sections of the Condensed Matter Division will be held by mail as soon as the preparations are complete.

Below is a provisional list of candidates prepared by the Chairman of the Division in consultation with the present Committee Chairmen. Individual Ordinary Members who are Section members and National Societies, Category $4 \mathrm{~b}$ ), are invited to make additional nominations. Those made by IOMs should be supported by at least six members.

Each Committee consist of six full members, elected for a term of three years, plus up to six co-opted members. The Sections then elect their own Chairmen and these serve on the Board of the Division. The maximum continuous period over which a Committee member may serve is six years.

In the lists that follow, the names of the Committee members elected at the last elections in 1983/84 are marked with an asterisk.

Liquids Section

S. Barocchi, Florence

S. Bratos, Paris

Th. Dorfmüller, Bielefeld

J. Janik, Cracow

P. Mazur, Leiden

G. Nicolis, Brussels

M.G. Velarde, Madrid

The above are the candidates put forward

by the Provisional Committee.

Low Temperature Section

*) Y. Bruynseraede, Leuven

S. Erne, Berlin (West)

J. Hook, Manchester

J.P. Laheurte, Nice

H.R. Ott, Zurich

G.L. Romani, Rome

Macromolecular Section

*) F.J. Baltà-Calleja, Madrid L.L. Chapoy, Lingby

*) H.G. Kilian, UIm R. Koningsveld, Sweikhuizen E. Martucelli, Naples

*) J.P. Mercier, Louvain-la-Neuve J.Spevacek, Prague

Magnetism Section J. Campbell, Orsay J.A. Mydosh, Leiden

*) J. Kaczer, Prague

*) E. Karlsson, Uppsala

*) S. Methfessel, Bochum P. Wachter, Zurich
Metals Section

*) D. Bacon, Liverpool

*) R. Cahn, Cambridge

*) E. Lüscher, Munich

Unless further nominations are received the Committee will cease to function at the end of its term.

Semiconductors and Insulators Section

*) J.W. Allen, St. Andrews

K.F. Berggren, Linköping

*) P. Gunter, Zurich

G. Martinez, Grenoble

M. Schultz, Erlangen

A. Stella, Pavia

B. Velicky, Prague

D.L. Weaire, Dublin

Surfaces and Interfaces Section

V. Bortolani, Modena

D. Echenique, San Sebastian

S. Holloway, Liverpool

B. Kasemo, Göteborg

G. LeLay, Marseille

F. Meier, Zurich

F. Netzer, Innsbruck

K.H. Rieder, Berlin (West)

Nominations should arrive at the EPS Secretariat by the end of May 1987 at the latest.
EPS Divisions, Sections and Group

Astronomy and Astrophysics Division Solar Physics Section

Atomic and Molecular Physics Division

Atomic Spectroscopy Section

Chemical Physics

Electronic and Atomic Collisions
Molecular Physics

Computational Physics Group

Condensed Matter Division

Liquids Section
Lionsed Matter

Liquids Section

Macromolecular Physics

Magnetism

Metal Physics

Semiconductors and Insulators

Surfaces and Interfaces

High Energy \& Particle Physics Division

Interdiv. Group on Exptl. Phys. Control Systems

Interdiv. Group on Physics for Development

Nuclear Physics Division

Optics Division

Plasma Physics Division

Quantum Electronics Division
Europhysics News is the official journal of the European Physical Society which comprises 29 National Socie ties, Academies and Group, about 4000 Individual Members and 70 Associate Members. Governing bodies of EPS are the General Meeting, Council and an dies of EPS are the General Meeting, Council and an policy. EPS promotes the collaboration of physicists throughout Europe, organising and harmonising confe rences and publications, improving physics education encouraging physics applications, awarding scholarencouraging physics applications, awarding schola publishes in addition to Europhys. News, Europhysics Letters (in partnership with national societies), European Journa of Physics (in partnership with The UK Inst. of Phys.) Europhys. Conf. Abs., Europhys. Ed. News. Individual Members receive Europhys. News free of charge (price to insts.: Sw.Fr. 90.-/a), Europhys. Lett. at Sw.Fr. 70.-1a (insts. 595.-1, rebates on many other publications and on conference fees. Annual EPS membership fee for In dividual Members belonging to an EPS member society is: Sw.Fr. 44.-; independent members: Sw.Fr. 132.members of a Collaborating Society: Sw.Fr. 55.

\section{Editor: E.N. Shaw \\ Editorial Board \\ A. Baratoff, F. James, M. Lehmann, \\ M. Mayor, J. Muller, M. Siegrist \\ Editorial and Advertising Office at the EPS Secretariat \\ Address: EUROPEAN PHYSICAL SOCIETY P.O. Box 69 $\mathrm{CH}-1213$ Petit-Lancy 2 Switzerland \\ Telephone: Geneva (22) 931130 \\ Telex : 428024 eps ch \\ Cables: europhys genève \\ Printed by: Pfirter frères sa CH-1213 Petit-Lancy/Switzerland}

